\title{
Knowledge Management: Hype, Hope, or Help?
}

\author{
David C. Blair \\ School of Business, The University of Michigan, Ann Arbor, MI 48109-1234
}

\begin{abstract}
This article examines the nature of Knowledge Management-how it differs from Data Management and Information Management, and its relationship to the development of Expert Systems and Decision Support Systems. It also examines the importance of Communities of Practice and Tacit Knowledge for Knowledge Management. The discussion is organized around five explicit questions. One: What is "knowledge"? Two: Why are people, especially managers, thinking about Knowledge Management? Three: What are the enabling technologies for Knowledge Management? Four: What are the prerequisites for Knowledge Management? Five: What are the major challenges for Knowledge Management?
\end{abstract}

\section{Introduction}

"Knowledge Management" has been a phrase in the vocabulary of management for some time now. There are articles written on "Knowledge Management," "Knowledge Management" journals, "Knowledge Management" conferences-both practical and academic-and to the ranks of DBAs (Data Base Administrators) and CIOs (Chief Information Officers) we have now added the CKO-the Chief Knowledge Officer. The idea of "knowledge" has always had an appealing cachet to it, although its saliency has been long associated more with universities and higher education than with the day-to-day toil of industry or the practice of management. Yet despite the persistence of the phrase "Knowledge Management" and the genuine feeling that "Knowledge Management" does refer to a new and legitimate practice, there is some ambiguity about what it really means, and little consensus about how to do it. The purpose of this discussion is to look at "Knowledge Management" carefully and try to understand what it is, or at least what it could be. The discussion will be organized around several questions: (1) What is "knowledge"? (2) Why are people, especially managers, thinking about Knowledge Management now? (3) What are the enabling technologies for Knowledge Management? (4) What are the prerequisites for

(c) 2002 Wiley Periodicals, Inc.
Knowledge Management? (5) What are the major challenges for Knowledge Management?

This is an ambitious agenda, but even tentative answers to these questions may go a long way towards helping us to understand whether Knowledge Management really is something we should pay attention to. In particular, is Knowledge Management really a legitimate and useful practice-is it something we can learn or teach, and, even more importantly, is it something that does any good to practice-does it make things better?

\section{What is "Knowledge"?}

Because we are already familiar with the practices of "Data Management" and "Information Management," it makes sense to ask whether we would we be doing anything differently if we did "Knowledge Management"? Whatever differences there may be in these practices must be due, at least in part, to the differences between Data, Information and Knowledge, so we will begin here. "Data" are clearly the easiest things to describe; they are simply "facts" and "figures" that are meaningful in some way. Typical items of data are account balances, demographic statistics, or names and addresses. Our daily newspapers are filled with data, from stock quotes to baseball statistics. There should be little disagreement about this category, even among individuals who do not deal with data on a regular basis. "Information," though a more complex notion than data, is not too problematic either. Peter Drucker's definition of "information" as "data that has been organized for a particular purpose" (Drucker, 1998) seems to capture the essential difference between data and information. Consider, for example, a list of the names and addresses of a company's customers. This is a collection of data, but because it is not organized for a particular purpose or use, we would probably not call it "information." To turn it into "information" we have to identify a particular use for it, and structure it so that it can be used as easily as possible. The names and addresses of customers have a number of uses, but salespeople use them primarily to contact current customers of a particular type. To this end, we might include in a customer list only those customers who have ordered something from 
the manufacturer within the last 6 months - in other words, they are active customers. Further, we might include data on what product or products they ordered within the last 6 months, to give salespeople some idea about individual customer's preferences. Data might also be included about how much total business they have done with the manufacturer, to give salespeople some idea about who the best of the active customers are. Finally, the customer list might be organized by sales region, so that salespeople can see more easily which customers are physically close to each other, or which are within a particular salesperson's exclusive region. It is just this organization of data for a particular use that turns it into information.

Although "data" and "information" are not difficult to define, "knowledge" is a more elusive concept. The antecedents of "knowledge" come from our intellectual history. The most unequivocal example of "knowledge" is probably philosophical knowledge. Philosophers from the ancient Socrates to today's Fred Dretske have tried to describe what "knowledge" is (Dretske, 1981). The accepted philosophical definition of 'knowledge" is "justified, true belief." Although such a definition is useful to philosophers, it has somewhat less utility for the knowledge manager, although the idea of "knowledge" as something whose truth you can justify or substantiate, does give us some separation from "data" and "information." That is, I can have "data" or "information" without having the ability to justify it myself - I simply accept it on good authority. To be "knowledgeable," though, often implies that an individual can justify or substantiate what he or she knows. But clearly "knowledge" in the organizational sense, which we are considering here, as something different from "data" and "information," is something more than simply "justified, true belief." But how can we bring this difference out? We can take a lesson from another philosopher who was often concerned about language and "what we mean" by certain words or phrases_-Ludwig Wittgenstein. Wittgenstein admonished us that if we really wanted to understand the meaning of a word or phrase, we shouldn't ask for the definition we should look at how it is actually used. We should "let the use teach [us] the meaning" (Wittgenstein, 1953). In other words, if we look at enough actual uses of a word, we will begin to see what it means (Blair, 1990; Blair, in progress).

The first thing that we might look at is whether "knowledge" is the same kind of thing that "data" and "information" are. Following Wittgenstein's example, we might hypothesize that if "data," "information," and "knowledge" are the same kind of things we could use the words interchangeably in specific phrases or sentences. For example, we might say:

"Put the data on the desk."

or,

"Put the information on the desk."

But would we ever say,

"Put the knowledge on the desk"?
The first two sentences are acceptable, but the third one seems quite odd. To bring this out more strongly we can use this substitution method in the following sentences:

"Get the data and fax it to New York."

"Get the information and fax it to New York."

But would we ever say,

"Get the knowledge and fax it to New York"?

Again, the first two sentences are fine, but the third one seems to be clearly unacceptable.

Often we speak of "having" data or information:

"Bill has the data you need."

"Mary has the information the CEO wants."

But what about,

"Chris has a knowledge of chemistry"?

Unlike the first two examples, here knowledge seems to fit the same pattern of usage as data and information. So we can say that we have knowledge in much the way that we can have data and information. But let's change the examples a little:

"Bill had the data, but he lost it."

"Mary had the information, but she misplaced it."

"Chris had the knowledge yesterday, but lost it."

Again, the third sentence will strike most readers as being unacceptable. Here we can see that although we can have knowledge it does not appear to be something that we can have in the same way that we have data or information. Knowledge is not something tangible that we can possess, exchange, or lose the way that we can with data or information. But what of the following sentence:

"I used to know how to tune an automobile engine, but I don't know how to do it any more."

This particular use of "know" seems OK, so there is something about knowledge that we can lose-that is, we can know something, and then some time later come to not know it. This brings out an essential difference between data, information, and knowledge, namely, that when we lose data or information, we often lose something that we can physically possess, something tangible. But when we lose knowledge, what we lose is an ability to do something. Abilities are not things that we can lose suddenly like data or information; we lose an ability more gradually over time as it falls into disuse. Philosophers bring out this difference by distinguishing between knowing how to do something, and knowing that something is the case. To know that something is the case is to possess data or information of a certain specific kind ('I know that the capital of California is Sacramento."). But to know how to do something is to possess an ability - to exercise a kind of expertise. We say that someone "knows how to speak German" or "knows how to pick stocks that are good investments." Of course, to exercise an expertise, you often need data or informationfor example, to pick good stocks you have to have current data or information about stock prices and their recent trends. But the data or information that enables a knowledgeable person to exercise some expertise is not sufficient by itself to enable someone else to exercise that expertise. That is, even if you have all the current information about 
stock prices and recent trends, it doesn't mean that you will now be able to pick good stocks for investment. Data and information may be necessary for the exercise of expertise but they are not sufficient for it. Knowledge Management, it seems, has two parts: first, there is the management of supporting data and information, and second, there is the management of a particular expertise, that is, the management of individuals with specific abilities.

From this discussion it should be clear that knowledge really is different from data or information. We can say:

A computer can have data (e.g., facts and figures stored in a data base).

A report can have information; that is, a report can be informative.

But only a person can be knowledgeable, that is, only a person can have and exercise knowledge.

\section{Context}

Another important distinction between data, information, and knowledge concerns "context." Data is relatively context free. For example, a name-and-address is a name and address no matter where it appears - in a memo, a report, a news article, or on the wall of a public phone booth. The context doesn't affect whether it is a name-and-address or not. But a name-and-address can be transformed into information by providing it with an interpretive context. For example, a name-and-address in a report listing active customers is not just a name-and-address, it is a name-andaddress of an active customer. The report provides a meaningful context for the name-and-address and gives some indication about how it can be used. But what about knowledge or expertise, how does that fit in here? First of all, we can say that it takes knowledge to turn data into information. Here, to come up with a report listing active customers, you would have to know how to determine whether a particular name-and-address is that of an active customer. This might involve examining orders that have been placed recently with the company, and then talking to salespeople to find customers who are considered active but have not ordered anything recently. In doing this, the knowledgeable person will have to draw on his or her experience to make judgments as to whether each name-and-address is that of an active customer or not. This is a process that relies less on specific quantifiable rules than it does on the individual's ability to make these judgments, and the ability to make these judgments relies, in turn, on an understanding of how the names and addresses of active customers are used.

But knowledge is often much more complex than simply determining which names and addresses are of active customers. Consider what it takes to know how to be a good salesperson. Salesmanship is a kind of expertise, and some individuals make better salespeople than others-some people know how to sell things better than others do. A knowledgeable salesperson possesses not only data and information, such as a list of active customers, but also has the experience to infer relevant things about customers that may not be immediately obvious to someone without that experience. For example, a knowledgeable salesperson might look at the address of an active customer and infer that because the address indicates that they live in a certain part of town, they are likely to be in a specific socio-economic class and thereby more willing to buy certain kinds of products rather than others. They might match a listing of the addresses of recently purchased homes with the list of customer addresses to find customers who have recently moved into a new home. With this information, they might infer that customers in new homes may be more interested in buying furniture and interior decorating supplies than other customers might be. Again, this is only an inference that a salesperson with relevant experience can make. Davenport's and Prusak's (1998) definition of "knowledge" captures this complexity:

Knowledge is a fluid mix of framed experience, values, contextual information, and expert insight that provides a framework for evaluating and incorporating new experiences and information. It originates and is applied in the minds of knowers. In organizations, it often becomes embedded not only in documents or repositories but also in organizational routines, processes, practices, and norms.

\section{Why Are People, Especially Managers, Thinking About Knowledge Management Now?}

There are several factors that have turned managers' attention to Knowledge Management. First of all, there is a realization that when an organization's employees "have knowledge" that is useful to the enterprise they possess something in addition to the data and information stored in the organization's information systems. It is this "additional something" that can leave the organization if the employee departs. Documentum's CEO Jeffrey Miller (Miller, 1998) expressed it well when he said:

Every afternoon our corporate knowledge walks out the door and I hope to God they'll be back tomorrow.

Although most employees who leave at night will come back, it is a fact of business in the United States that employees change jobs frequently, and, it is rare that a worker will spend his or her entire career with one organization. Americans are a very mobile society, and have had a history of being willing to move to take advantage of better prospects - the vast majority of Americans are descended from immigrants who did precisely that. But there is another, more recent, dynamic that has increased this mobility. As analytical skills become more valuable for organizations, the relatively stable layers and skills of middle management are being replaced by more autonomous managers with strong analytical skills. These workers are often more loyal to their skills or expertise than to the organizations for which they work (Stewart, 1997). This means that they are more attracted to opportunities to use 
their skills than they are to working for a single organization. This is a phenomenon that has characterized the more technical vocations such as computer programming, data base management, and telecommunications, whose practitioners have always been highly mobile. The difference today is that strong analytical skills are being required of all workers and managers, not just those in the more technical areas or industries. Economist and former Secretary of Labor Robert Reich has written convincingly of the reasons for this change (Reich, 1991). In short, U.S. industries that had competed by producing high volumes of products at low cost can no longer compete in this way against the rising industrialization of Third-World countries that have vastly cheaper work forces. Now that much current manufacturing technology is widely available, Third World countries can easily produce products more cheaply than we can. Reich insists that the only kind of competition that the United States can successfully sustain is to produce products or services that are either innovative or tailor made for individual customers. This kind of competition requires a highly educated workforce with strong analytical skills. As the value of an employee becomes more dependent on his or her analytical skills and expertise, and less dependent on longevity with one company, employees will naturally move to the organizations that offer them the best opportunity to use and develop their expertise. Because skill acquisition and maintenance are ongoing processes, it stands to reason that as an employee's expertise gets better he/she may need to change organizations to find opportunities to utilize or improve those skills.

Knowledge Management is not so much the management of tangible assets such as data or information, but the active management and support of expertise. This is not to say that there are no tangible assets that need to be managed in Knowledge Management, but to say that access to these assets alone is not sufficient to guarantee successful execution of expertise.

\section{Knowledge Management and Communities of Practice}

Now if Knowledge Management is largely the management and support of expertise, then, unlike data and information management, it is primarily the management of individuals with specific abilities, rather than the management of repositories of data and information. This has some interesting consequences for management. In the first place, the "knowledge" that is managed is not easily separable from the individuals who exercise it - the practitioners themselves become the "repositories" for knowledge. How, then, do you pass knowledge from those who have it to those who do not? Because Knowledge is not something that can usually be written down, knowledgeable individuals must be encouraged to pass their expertise to others through personal contact. This is a very interactive process, and often takes the form of a kind of apprenticeship with novices interacting closely with experts to "learn their trade." This means that Knowledge Management must in large part be concerned with establishing, maintaining, and facilitating communication between both experts and novices (experts to experts, experts to novices, and novices to novices). This is where the notion of a "Community of Practice" arises (Davenport \& Prusak, 1998). Again, there may be some ordinary data or information management needed to support this process, but neither data nor information management by itself is sufficient to enable individuals to exercise some expertise.

One of the interesting consequences of establishing and maintaining communities of practice is that it requires one major commitment on the part of its members - the experts and novices must be willing to share what they know with others. This requirement, though, can pose problems in many of today's organizations. Specifically, if an employee becomes valuable to the organization because of certain specific skills and abilities that he or she can exercise, then there is often a powerful incentive for that employee not to pass that expertise on to others. That is, by helping other employees to become more expert in a particular area, an expert loses the exclusivity of his or her expertise. A prominent consulting company came face to face with this problem: when they found that their senior consultants were not talking to or interacting with their junior or entry-level consultants, they offered bonuses to the senior consultants as an incentive to interact with the junior consultants. This had little effect. The company then had to threaten the senior consultants with the loss of earned bonuses if they did not increase their communication with junior consultants. Of course, this raises a lot of management issues, not the least of which is how do you determine whether the communication between senior and junior practitioners really does help the junior practitioners to get better, that is, to become more knowledgeable? Some companies have begun monitoring e-mail and phone calls to determine how much communication is taking place between those who have an important expertise and those who do not. But a hundred e-mail messages may be less helpful than the tip an expert might give a novice when they meet at the water cooler and discuss their work informally.

Shell Oil Company considers its paleontologists to be some of their most valuable experts, and has introduced a formal system to encourage the meaningful exchange of knowledge between their senior and junior paleontologists (McDermott, 1998). Shell paleontologists are required to meet regularly, and to present the details of whatever project they are working on to the other paleontologists. This allows the novice paleontologists to see how the expert paleontologists approach a problem, and, when the novices present their work, it enables them to get feedback on what they are doing from those more expert than they are.

Unless novices can get all of their expertise out of book (and this is not likely to be the case in most areas of expertise), they will have to develop their abilities through the interaction with experts in their area. Organizations, like Shell Oil Company in the above example, can do some 
things to facilitate the sharing of expertise between experts and novices. But there must, as we said, be a willingness on the part of the experts to pass their expertise-their knowledge-to those who are less expert than they are. It is well known among organizational theorists that organizations have a kind of character or ambience about them, what is usually called their "culture." Schienstock gives us a more precise definition: "[organizational culture is] . . . a network of interlocking rituals, norms, assumptions, and values that have developed out of continuous interactions among the members of an organization" (quoted in Cronin, 2001). The kind of culture of an organization can be an important factor influencing whether it encourages the sharing of knowledge between employees or inhibits it. Organizations that have a culture of being very competitive internally, with employees competing against each other for customers and projects, will have a great deal of trouble convincing expert employees to pass their expertise/knowledge on to those who are less knowledgeable. Unfortunately, it may be very difficult for an organization whose culture is not conducive to sharing to transform itself into an organization that encourages sharing. Organizational theorists are pretty much in agreement that one of the hardest things to change about an organization is its culture (Trice \& Beyer, 1993).

\section{The Diminishing Returns of Information/Data Management}

A number of other factors have contributed to the emergence of Knowledge Management. First of all, Knowledge Management must be seen, not as a phenomenon without precedent, but as a process that has been heavily influenced by the growth and application of computer technology to data and information management. The earliest applications of computer technology in business were the automation of what before were manual processes: payroll, inventory control, ordering, billing, etc. These were not only the first applications, they were the most clearly successful, from a financial point of view. By automating manual information processes, organizations were able to gain a clear return on their investment in computer technology: the cost of buying, installing and maintaining computers could be compared to the explicit savings gotten by laying off the salaried employees who carried out these tasks. These early business applications of computers came to be called 'transaction processing systems" (TPSs) because they managed the transactions of the organization. Early TPSs were conceptually simple and limited in the kind of information they provided, primarily lists or summaries of transactions. This changed with the development and introduction of data base management technology in the 1970s and 80s. Data base management systems, especially ones based on the relational model, allowed the users of TPSs to arrange, quickly and easily, the data/information provided by these systems in new and creative ways. This flexibility in arranging and presenting data and information enabled the users of these systems to discover correlations in the data that they had not seen before (e.g., finding that the sales of a particular product are strongly correlated to specific times of the year, specific geographical regions, or the sales of other products). But as computer technology became ubiquitous, all but the smallest organizations automated their fundamental transaction processes, and as data base management systems became cheaper and widely available the advantages of flexible data and information management also became widespread throughout industry. Naturally, as computer technology spread, the advantage of having automated transaction processing systems and data base management systems spread to the point where the technology conferred no real advantage to its users, it was simply a cost of doing business.

Naturally, there have been continuing attempts to leverage further advantage out of computerized TPSs, in particular, by making them the foundation for higher level systems such as decision-support sytems and expert systems. But although the advantage conferred by automating transaction processing systems was clear and unequivocal, neither decision-support systems (DSSs) nor expert systems (ESs) have provided a clear positive return on investment (there are, of course, some successful DSSs and ESs, but they are rare and the overall success of DSSs and ESs pales in comparison to the successful application of earlier computer technologies such as data base management, telecommunications, and the automation of transaction processing). Some analysts have declared DSSs a resounding failure (Kelly, 1996), and others have offered convincing arguments for the impossibility of any widespread ES development (Dreyfus \& Dreyfus, 1986). Even history offers an apropos analogy. In the California Gold Rush of 1849, vanishingly few individuals struck it rich, and the only individuals sure of profiting from the quest for gold were those who sold the equipment that the miners used. Likewise, the only individuals to profit consistently from the rush to build Expert Systems in the late 1980s and 1990s were companies like Symbolics, who sold the software and hardware to build Expert Systems (Blair, 1985). Like the number of gold miners who struck it rich in California, the number of commercially successful ESs is vanishingly small. The specific reasons for the failure of DSSs and ESs to attain any general level of success are beyond the scope of this article, but one reason deserves mentioning. Decision Support Systems and Expert Systems attempt to improve human decision making (DSSs) or replace it entirely (ESs). But human decision making is a kind of expertise, it is the activity of knowledgeable individuals, and this expertise is not something that, in general, can be easily supplemented or replaced by computer technology, as DSSs and ESs tried to do. In contrast, Knowledge Management does not try to actively supplement or replace human expertise, it simply tries to encourage and facilitate it. For DSSs and ESs, the expert was external to the system, but for Knowledge Management, the expert is an essential part of the system.

Other, more recent efforts to leverage further advantage from Transaction Processing Systems are Data Warehous- 
ing, Data Mining, and Enterprise Resource Planning. There have been successes in these areas, but, again, nothing of the scale of success enjoyed by the earlier applications of data base management, telecommunications, and the automation of TPSs, and, most importantly, many successful businesses continue to do well without any efforts in these areas. In contrast, few sizable businesses could exist without automated TPSs, telecommunications facilities, and data base management systems. The continuing attempts to leverage further advantage from TPSs stem from the belief that there is more information in them than what has been delivered so far.

The emergence of Knowledge Management is, in some sense, the consequence of, first, the realization that there is "something more" to be extracted from current information/ data systems than what is actually stored on them, and, second, the poor record of DSSs and ESs in supporting, capturing or utilizing this additional information.

In particular, ES development comes in large part from the attempt to capture in an information system something that cannot be captured by it-knowledge.

\section{The Hope and Promise of Automation}

There is no doubt that the progress we have made in automating the storage and transmission of written information has been remarkable. We can make multiple copies of the longest document and e-mail it to myriad addressees with a few keystrokes. We can store the contents of 50 filing cabinets in a laptop computer or on a removable hard disk that can fit in our pocket. But this rapid development of storage technology has not come without consequences. When information is put on a computer it is usually removed from the informal social networks of the employees who use and produce it. Removing information from these pragmatic contexts is $\mathrm{OK}$ for relatively context-free data, and low-context information, but it can be harmful to remove the more context-dependent information that experts often use in the execution of their expertise. Seen this way, the emergence of Knowledge Management is, in part, a consequence of the increasingly pervasive automation of an organization's information. Documents, which are often described as an important component in Knowledge Management, are a good example. When documents exist only as paper, they tend to be physically managed by individuals who understand them and have some use for them. These individuals provide a number of important functions for this information: first, they can serve as "gatekeepers" for the documents they possess; that is, they can direct inquiring individuals to the documents that they are most likely to need. Because the gatekeepers are usually individuals who use, or have used, the information that they possess, they are also usually able to interpret or explain the meaning of that information, that is, how it is used by the organization. Second, if they do not have the documents an inquirer wants they can often direct him/her to another gatekeeper who is more likely to have them. Finally, because the storage of paper documents has an explicit cost, the gatekeepers will be selective about what they keep, and will often weed out and discard documents that are no longer useful. This makes it more likely that social information networks will provide access to information that is useful for the purposes of the organization. Although these social information networks are informal in nature, they can be quite efficient in providing precise access to a large body of information.

Paper-based documents are frequently associated with messy offices populated by desks and tables piled with stacks of papers. As humorous as this image is, there is a hidden efficiency in it. Surprisingly, studies have shown that messy offices can be surprisingly efficient mechanisms for arranging information (The Economist, 1992; Landale \& Edmonds, 1992; Lansdale, Young, \& Bass, 1989).The piles of documents can be moved easily, and the documents themselves can be grouped and regrouped to facilitate different conceptual arrangements of the information. The spatial arrangement tends to be implicitly value-based also, with the importance of the documents decreasing in direct proportion to their distance from the part of the office where most of the work gets done, usually the desk. This sort of easy grouping, regrouping, and value-based arrangement of documents is very difficult to duplicate in an computerized retrieval system, and virtually impossible in systems that simply list files sequentially with only a brief description of their contents.

If we could capture knowledge in its entirety in some kind of tangible form, Knowledge Management would be far more straightforward-we would simply store knowledge and manage it much like we manage information and data. One of the assumptions of the Fifth-Generation project in Artificial intelligence (the attempt to commercialize advances in $\mathrm{AI}$ ) was that any human skill or expertise that could be performed consistently could ultimately be completely described by a set of rules or procedures (Feigenbaum \& McCorduck, 1983, 1988). As attractive as this assumption is, there is much evidence that in most areas of expertise, it does not hold. As philosopher of science Hilary Putnam put it, "it is a mistake to think that merely because one practices an activity one can give a theory of it" (Magee, 1997, p. 235). The assumption that human expertise can be completely described by rules, procedures, and relevant facts, has been an implicit assumption behind much of the work in Expert Systems. But in their critique of Expert Systems development, Dreyfus and Dreyfus (1986) showed that for most areas of expertise, rules, procedures, and facts can, at best, only capture rudimentary levels of expert behavior-what they call "competence." Competence is only the third stage, out of seven, in the acquisition of expertise. As Dreyfus puts it, “. . . expert systems following rules and procedures... would at best be competent" (Dreyfus, 2001, p. 48). But most organizations cannot be built on just competence. It is a fact of life that for an organization, especially a business, to survive it must bring the highest levels of expertise to bear on the activities and practices in which it is engaged. Expert Systems that mod- 
eled only competent behavior have little chance of commercial success.

Another difficulty with the Artificial Intelligence model of expert behavior is that it assumes that the controller of the behavior is a single, centralized entity like the mind. But some workers in Robotics and Neuroscience have called this assumption into question. As Clark (1997) puts it:

\begin{abstract}
The New Robotics revolution rejects a fundamental part of the classical image of mind. It rejects the image of a central planner that is privy to all the information available anywhere in the system and dedicated to the discovery of possible behavioral sequences that will satisfy particular goals. The trouble with the central planner is that it is profoundly impractical. It introduces what Rodney Brooks aptly termed a "representational bottleneck" blocking fast, real-time response. The reason is that the incoming sensory information must be converted into a single symbolic code so that such a planner can deal with it. And the planners' output will itself have to be converted from its proprietary code into the various formats needed to control various types of motor response. These steps of translation are time-consuming and expensive.
\end{abstract}

The inability of Expert System development to model, in most cases, more than basic competence has been one of the major factors in the failure of this application of technology. The importance of this for Knowledge Management is clear: if knowledge is primarily expertise, and expertise is not something that we can model and separate from those who practice it, then Knowledge Management cannot operate apart from the experts who practice a particular expertise.

\section{The Question of Tacit Knowledge}

One issue that arises frequently in Knowledge Management is the notion of "tacit knowledge." Tacit knowledge is assumed to be what the practicing expert knows-it is what enables him or her to do what they do. There are two kinds of tacit knowledge, though: that which has not been expressed but is potentially expressible, and that which is not expressible. As an example of the first type, consider a construction engineer putting a particular kind of brace into the structure supporting the roof of a building she is erecting. Although she has selected a particular kind of brace based on her experience doing this sort of thing many times, it may be possible to write down a rule or procedure that could be used by a novice engineer, or an engineering student, to select the proper kind of brace for this job. As an example of the second type of tacit knowledge consider what the wine connoisseur knows. In this case, what the wine connoisseur knows when he selects the best available wine is not reducible to a rule or set of rules- he simply knows the difference between good and not-so-good wine, and would be at a loss if required to state the precise reasons one wine is better than another. This kind of tacit knowledge is only expressible by demonstration - the wine connoisseur can show you a good and a not-so-good wine without being able to describe why one is good and the other is not, except in the vaguest possible ways-ways that could only be understood by other wine connoisseurs. This is why aspiring wine connoisseurs do not read books about their craft, they attend wine-tasting parties hosted by experts. Even Mark Twain (1965) saw the tacit dimension of expertise. Consider the following passage from his semi-autobiographical Life on the Mississippi, where he describes a critical event in his training as a river pilot (in this passage, the pilot has just ordered Twain, the apprentice pilot, to steer the boat over what Twain thinks is a deadly reef which will sink the boat):

[Twain] [we] made a straight break for the reef. As it disappeared under our bows I held my breath: but we slid over it like oil.

[Pilot] Now don't you see the difference? it wasn't anything but a wind reef. The wind does that.

[Twain] So I see. But it is exactly like a [real] reef. How am I ever going to tell them apart?

[Pilot] I can't tell you. It's an instinct. By and by you will just naturally know one from the other, but you never will be able to explain why or how you know them apart.

It turned out to be true. The face of the water, in time, became a wonderful book-a book that was a dead language to the uneducated passenger, but which told its mind to me without reserve, delivering its most cherished secrets as clearly as if it uttered them with a voice. And it was not a book to be read once and thrown aside, for it had a new story to tell every day.

What Dreyfus and Dreyfus' work (1986) has shown is that most expertise is of the latter kind, it is, in whole or in part, inexpressible, and even the expressible tacit knowledge, such as that possessed by the engineer, when it is written down only expresses at best a merely competent level of expertise. But the experienced engineer is not just competent, she is able to select the correct brace to use in all the ordinary construction situations-the "textbook" cases-but can also select the right brace in situations that are unusual or unique, situations in which the novice engineer with just the rules will be at a loss.

Dreyfus and Dreyfus (1986) do not say that there is nothing tacit involved in expertise. They are only saying that the assumption that every expert has some tacit set of rules that completely describes his or her expertise is erroneous. Instead of rules, experts often have heuristics-rules of thumb-that they find useful in the exercise of their abilities. Such heuristics, although not completely describing the execution of an expertise, are often useful to other experts or to novice experts. Capturing such heuristics, when available, can be a useful part of Knowledge Management.

If most expertise cannot be reduced to a set of rules that completely defines it, then the best source of information 
about what experts do are the descriptions of the actual "cases" in which those experts utilize their expertise. These case descriptions would include a statement of the problem being solved, the circumstances that are relevant to the case, the steps the expert goes through in the solution of the problem, the specification of useful data or information relevant to the expertise, and the outcome. The cases that have been executed most successfully can form the foundation for the establishment of a set of "best practices." Best practices can serve a number of purposes: they can be used to form a benchmark for the level of quality expected of practicing experts; they can also be arranged in order of increasing complexity or difficulty to form a set of graduated examples useful for the training of novices. The gathering of Best Practices is often an important component of Knowledge Management (Davenport \& Prusak, 1998).

\section{What Are the Enabling Technologies for KM?}

Although Knowledge Management is not the same as data or information management, data and information retrieval can be important components of it. But just as knowledge and expertise can be unpredictably varied, the data and information that assist them can be unpredictably variable in their form or content. Data, text, images, schematics, video, audio, Web pages, compound and multimedia documents can all be important ancillaries to Knowledge Management. Consequently, we must have widely available commercial technology that can physically manage this wide variety of informative media. Fortunately, we do have this-Oracle's 9i Data Base Management System that appeared recently is a good example of the technology that can manage this wide variety of informative media that may be necessary for Knowledge Management. Other data base vendors, like IBM and Informix, have similar technologies.

Because a wide variety of media may be relevant to Knowledge Management, it is clear that we will also need to access these media in a wide variety of places and through a variety of networks, storage systems, user platforms, and interfaces. Consequently, we will need communication networks that can handle this variety. We need cheap, highbandwidth communication channels, and a common multimedia communication protocol that will allow a wide variety of systems to communicate with each other. Again we are fortunate - the internet has both a high enough bandwidth and a communication protocol (TCP/IP) that is freely available and widely distributed. As users have come to insist on higher and higher bandwidth to gain access to more complex information, communications engineers have struggled to increase the bandwidth of the "last mile" that connects many users to the higher bandwidth internet backbones. Again, we are fortunate. With technologies like the earlier ISDN (Integrated Services Digital Network) and the more recent DSL (Digital Subscriber Line) engineers have increased the capacity of our installed base of "twisted pair" copper phone lines to carry large amounts of complex information media into the home or small office. Until these advances, it was thought that ubiquitous high bandwidth could only come through the widespread installation of fiber optic connections. This would be both time consuming and prohibitively expensive to do on a large scale because it costs as much to "light" fiber optic lines as it does to install them, and installation alone was estimated to be in the $100 \mathrm{~s}$ of billions of dollars if every U.S. home and small business was to have it. It is true that ISDN and DSL are not available everywhere because they require the close proximity of special facilities. But as time passes DSL and ISDN are becoming increasingly widely available (the phone companies are the principal providers of this technology). Until then, cable TV connections can offer a high bandwidth digital service alternative to most who want it.

Another component for high bandwidth communication is a common, widely available multimedia markup language, so that complex information media can be represented in a way that can be understood by a wide variety of platforms and user interfaces. HTML and XML fit these requirements well.

Finally, the ability to store and transmit a wide variety of information types and formats in support of knowledge workers is of no use unless the individuals who want it can find it. This is probably the weakest link in Knowledge Management. As we put more information on the World Wide Web and on organizational intranets, we narrow the bandwidth of how we can express what we want. Current search engines have to base their searches primarily on the literal content of the information itself. in other words, the searcher must anticipate, in his search queries, the words and phrases that will appear in the documents he would be interested in. Yet this is a skill that we are not very good at (Blair \& Maron, 1985). To understand how limiting this can be, imagine the difference between using a search engine to find what you want and asking a colleague or professional searcher for the same information. When talking to an individual we can describe what we want in all the subtleties of our native language, while with the search engine we are limited to, at best, a kind of Boolean "pidgin-English."

It is clear that most of the enabling technologies for Knowledge Management are already here, having come about with the growth and development of the internet. Knowledge Management is, in one sense, a means of leveraging more advantage from the internet and the World Wide Web.

\section{What Are the Prerequisites for KM?}

A number of things have to happen before Knowledge Management can have a reasonable chance of success. In the first place, because Knowledge Management deals largely with practicing experts, and these experts must communicate with each other to improve their abilities and to train novices, the organization must have a culture that encourages and facilitates the sharing of expertise.

One of the basic principles of management is that you can't manage what you don't know about. This means that 
an essential prerequisite for Knowledge Management is to have some kind of "Knowledge inventory" or "Knowledge Map" that provides an explicit tabulation of the organization's knowledge assets. Because knowledge is strongly associated with individuals, a Knowledge inventory or Map will consist, in part, of a listing of individuals and their respective expertise. Included in this tabulation are not just a naming of these expert abilities, but a description of the kinds of problems these experts can solve. Businesses can take a lesson from universities, which expend a great deal of effort and resources describing the expertise and knowledge of their faculty and research associates.

Knowledge Management requires some significant changes in the abilities of what Peter Drucker called "Knowledge Workers" (Drucker, 1998). Because the information or data relevant to Knowledge Management may come from an unpredictable variety of sources, many of them outside of the organization, Knowledge Workers must have strong critical thinking skills; in particular, they must have a well-developed ability to evaluate the validity and reliability of information obtained from unfamiliar sources. The World Wide Web is a treasure of valuable information, of course, but it is also a landfill of inaccurate, incorrect, and sometimes fraudulent information also. Knowledge Workers, more than most other information dependent workers, are particularly reliant on the quality and accuracy of information from the Web.

One of the obvious goals of Knowledge Management is to create value within organizations, and one of the most common ways for Knowledge Workers to create value is to provide support for effective decision making - the kind of "analytical skill" that Robert Reich (1991) has stated is so valuable to the modern organization. ("Creating value" does not necessarily mean creating economic value. Nonprofit organizations, government organizations and charities create value in non-economic ways, they create what is now called "social capital.") But the kind of decision making that has been traditionally associated with organizations has been a very structured, logical process whose inputs and decision points can be defined very precisely. But the context of decision making for today's Knowledge Worker is much more complex-relevant information comes from a number of sources, many of which are outside the organization. Gary Klein calls this kind of decision context "naturalistic decision making," and describes its characteristics as including "time pressure, high stakes, inadequate information (information that is missing, ambiguous, or erroneous), ill-defined goals, [and] poorly defined procedures ..." (Klein, 1998). Having Knowledge Workers who can create value through effective decision making in this kind of environment is another prerequisite for Knowledge Management.

\section{What Are the Major Challenges for KM?}

As we have said, a prerequisite for effective Knowledge Management is an organizational culture that supports and facilitates the sharing of knowledge. But not all organizations have such a culture of sharing. Unfortunately, organizational culture is one of the hardest things for an organization to change, and there is no consensus on how to even attempt such a change (Trice \& Beyer, 1993). This means that organizations that do not have a culture of sharing face less than auspicious prospects for establishing effective Knowlege Management. It does not mean that Knowledge Management is impossible, but it does mean that it will be difficult, and its results more modest than those organizations with cultures of sharing.

Although we do have some understanding about how individuals become experts (Dreyfus \& Dreyfus, 1986), and some researchers have begun to look at the dynamics of communities of practice in organizations (Wenger, 1998; Wenger \& Snyder, 1999), there remains much to learn about these activities. But whatever we learn about how individuals attain expertise, and how they interact with other experts, will probably have a direct positive effect on the practice of Knowledge Management.

The treatment of Tacit Knowledge will continue to be a challenge for Knowledge Management. In particular, it will be important to make a clear distinction between that part of expertise that is unexpressed but expressible and that part of expertise that is simply inexpressible. Much of the history of Expert System development has been a saga of wasted time, effort, and money trying to make inexpressible expertise explicit-over a number of years, the Department of Defense funded some 300 expert systems projects at a cost of millions of dollars, yet only one of those projects was successful, a rate of success comparable, as we pointed out, to the California Gold Rush (H. Dreyfus, personal communication, 1999).

Finally, there is an important and complex legal issue that lurks within the practice of Knowledge Management: the nature of intellectual property. An expert certainly knows something when he or she can successfully exercise an expertise, but it is not at all clear precisely what it is of what the expert knows that belongs to the expert. This is an even greater issue when there is more than one expert and they interact frequently in the practice of their expertise. Independent consultants have had to deal with this issue when they work for organizations - they are often required to sign a contract that is quite explicit about what they bring to the organization and what they can use or divulge after they leave the organization. This is not a simple process even when an outside consultant brings an expertise that the organization does not have. But in the practice of Knowledge Management the organization usually deals with experts who are full-time employees of the organization and who may have acquired their expertise during their time with the organization. Here it is not at all clear what the expert "owns" and what he knows is the organization's property. Intellectual property law can take a lesson from copyright law. Copyright law was established explicitly to encourage the sharing of the public knowledge, not to restrict it. That is, it is only when an author's rights to what 
he or she has written are protected that the author will be willing to have his/her work published and distributed. In the same way, experts will be willing to share their expertise only insofar as what they "own" can be protected or compensated for. Obviously, what counts as intellectual property is far more complex and ambiguous than what can be copyrighted, but if sharing what experts know is as important for Knowledge Management as we have claimed here, then the specification and protection of both the expert's and the organization's intellectual property will be a sine qua non for the sharing necessary to Knowledge Management.

\section{Conclusion}

The title of this discussion asked whether Knowledge Management is "Hype, Hope, or Help?" It is not an equivocation to conclude that, in various manifestations, it can be all three. It has certainly been "hyped" beyond its ability to deliver clear benefits in all applications. Some of Knowledge Management has been simply an enthusiastic renaming of existing management practices: as the interest in Knowledge Management began to grow, the established industry publication "Image World" was renamed "KM World," reflecting the early association of Knowledge Management with the information that was kept as images: primarily pictures, drawings, and text. Knowledge Management has been variously associated with Document Management/Retrieval, and, currently, the management of expertise. The current work on the care, feeding, and training of experts seems to have identified a legitimate and promising area of management study - the work on "communities of practice" and "best practices" have certainly helped us to understand and better manage this valuable organizational resource, and there is certainly the hope that more study in this area will enable us to manage and leverage expertise better than we now do.

But in the final analysis, Knowledge Management is not an end in itself, it is a means to a further end. For the goal of Knowledge Workers is not so much to "manage knowledge" but to solve problems. As Thomas Huxley put it over a century ago: "The great end of knowledge is not knowledge but action."

\section{References}

Blair, D.C. (1985). Expert systems: The new gold rush! ASIS Midyear Meeting, Ann Arbor, MI.

Blair, D.C. (1990). Language and representation in information retrieval. Amsterdam: Elsevier Science.

Blair, D.C. (in progress). Wittgenstein, Language and information-"Back to the rough ground!"
Blair, D.C., \& Maron, M.E. (1985). An evaluation of retrieval effectiveness for a full-text document retrieval system. Communications of the ACM, 28(3), 289-299.

Clark, A. (1997). Being there: Putting brain, body, and world together again. Cambridge: MA: MIT Press.

Cronin, B. (2001). Knowledge management, organizational culture and Anglo-American higher education. Journal of Information Science, 27(3), 129-137.

Davenport, T., \& Prusak, L. (1998). Working knowledge. Boston: Harvard Business School Press.

Dreyfus, H. (2001). On the Internet. New York: Routledge.

Dreyfus, H., \& Dreyfus, S. (1986). Man over machine: the power of human intuition and expertise in the era of the computer. New York: The Free Press, Macmillan, Inc.

Dretske, F. (1981). Knowledge and the flow of information. Cambridge, MA: MIT.

Drucker, P. (1998). The coming of the new organization. In Harvard business review on knowledge management. Cambridge, MA: Harvard Business School Press. (Reprint of the article from the Harvard Business Review, January-February, 1988).

The Economist. (1992, September 5). Organizing offices: under the volcano. p. 91.

Feigenbaum, E., \& McCorduck, P. (1983). The fifth generation: Artificial intelligence \& Japan's computer challenge to the world. New York: Addison-Wesley Longman.

Feigenbaum, E., \& McCorduck, P. (1988). The rise of the expert company: how visionary companies are using artificial intelligence to achieve higher productivity \& profits. New York: Crown Publishing Group.

Kelly, S. (1996). Data warehousing: the route to mass customization. New York: John Wiley \& Sons.

Klein, G. (1998). Sources of power: How people make decisions. Cambridge, MA: MIT Press.

Lansdale, M.W., \& Edmonds, E. (1992). Using memory for events in the design of personal filing systems. International Journal of Man-Machine Studies, 36, 97-126.

Lansdale, M.W., Young, D.R., \& Bass. C.A. (1989). MEMOIRS: A personal multimedia information system. In A. Sutcliffe \& L. Macaulay (Eds.), People and computers V (pp. 315-330). Cambridge: Cambridge University Press.

Magee, B. (1997). Men of ideas. New York: The Viking Press.

McDermott, R. (1998). Learning across teams: How Shell Oil Co. uses learning communities to preserve technical excellence. DCI's Knowledge Management Conference Proceedings, November 3-5, 1998, Chicago, II.

Miller, J. (1998). Keynote address, DCI Conference on Knowledge Management, November 3-5, 1998 Chicago, II.

Reich, R. (1991). The work of nations: preparing ourselves for 21st century capitalism. New York: Alfred Knopf.

Stewart, T. (1997). Intellectual capital: the new wealth of organizations. New York: Doubleday.

Trice, H., \& Beyer, J. (1993). The cultures of work organizations. New York: Prentice-Hall.

Twain, M. (1965). Life on the Mississippi. New York: Harper and Row.

Wenger, E. (1998). Communities of practice: Learning, meaning and identity. Cambridge: Cambridge University Press.

Wenger, E.C., \& Snyder, W.M. (1999). Communities of practice: the organizational frontier. Harvard Business Review, 78(1) 139-145.

Wittgenstein, L. (1953). Philosophical investigations. Trans. by G.E.M. Anscombe. Oxford: B. Blackwell. 